\title{
INCONSCIENTE COLETIVO CIBERNÉTICO: SINGULARIDADE TECNOLÓGICA NA ERA DA INTERNET DAS COISAS
}

Eduardo dos Santos Pereira

\begin{abstract}
Possui graduação em Física pela Universidade Federal de São João Del-Rei (2005). Mestrado em Astrofísica pelo Instituto Nacional de Pesquisas Espaciais (2008). Doutorado em Astrofísica pelo Instituto Nacional de Pesquisas Espaciais (2012). Pós-doutorado em astrofísica pelo Instituto Nacional de Pesquisas Espaciais com bolsa FAPESP. E Pós-Doutorado em Astrofísica Observacional na USP (2017). Atuando principalmente nos seguintes temas: Cosmologia, Ondas Gravitacionais, Astrofísica Computacional e Astronomia Extragaláctica, Processamento de Imagens, Inteligência Artificial. E-mail: eduardo.santos@inpe.br
\end{abstract}

\section{RESUMO}

Internet das Coisas (Internet of Things - IoT) vai muito além da conexão de equipamentos diversos, como lâmpadas e sensores de temperatura, ou a rede mundial de computadores. Uma das grandes visões ligadas a essa tecnologia está na possibilidade de usar o fluxo de dados desses equipamentos para gerar sistemas que tomam decisões de forma autônoma. Como por exemplo, uma geladeira conectada a rede poderia fazer compras de forma automática, baseada nos costumes de uma família. Por outro lado, uma perspectiva interessante é de que num futuro relativamente próximo, devido ao avanço acelerado do desenvolvimento tecnológico, uma superinteligência artificial poderia emergir. Esse ponto de ruptura é conhecido como singularidade tecnológica. Neste contexto, o presente artigo é apresentado na forma de um ensaio teórico, o qual busca refletir em como a IoT poderia alcançar a singularidade tecnológica e como isso implicaria na redução do livre arbítrio a nível de sociedade.

PALAVRAS-CHAVE: Internet das Coisas. Singularidade Tecnológica. Inteligência Artificial.

\section{UNCONSCIOUS CYBER COLLECTIVE: TECHNOLOGICAL SINGULARITY IN THE AGE OF THE INTERNET OF}




\title{
THINGS
}

\begin{abstract}
Internet of Things (IoT) is much more than Internet connecting devices, like temperature sensors or lamps. An important vision concerning this technology, it is the possibility of using the information data flux from connected devices to make a real time automatic decisions. For example, a connected refrigerator could shop from online market, based on a family behaviour. On the other hand, some author consider that, in the near future, a superintelligence could arise from the accelerated technological development. This fact is known as technological singularity. In this article, a reflexion about the role of IoT to achieve the technological singularity is presented.
\end{abstract}

KEYWORDS: Internet of Things. Technological Singularity. Artificial Intelligence.

\section{INTRODUÇÃO}

Nos últimos anos, vimos um avanço acelerado do desenvolvimento tecnológico, o que alavancou a área de Inteligência Artificial e Computacional (IAC). Segundo Walsh (2017), esse crescimento despertou preocupações entre especialistas e pessoas de renome na área tecnológica. Como exemplo, o autor cita declarações do físico Stephen Hawking sobre os impactos negativos de um sistema de IAC. A maior preocupação é que o avanço acelerado poderia chegar a tal ponto que um sistema artificial pudesse evoluir de forma autônoma, superando e controlando a humanidade. Esse ponto de transformação é conhecido como singularidade tecnológica.

$\mathrm{Na}$ visão popular, a singularidade tecnológica ocorreria quando máquinas passam a ganhar consciência dominando a humanidade. Walsh (2017) também apresenta vários pontos contra esse tipo de singularidade. Entre eles, o autor indica que existe uma confusão entre o conceito de inteligência para fazer tarefas e a capacidade de aperfeiçoar a própria inteligência para fazer tarefas. Os sistemas de IAC atuais são extremamente especialistas, capazes de traduzir textos ou fazer reconhecimento de imagens. Porém, esses sistemas são incapazes de fazer uma autoconversão para aprender outras especialidades. Por outro lado, uma superinteligência artificial não seria a única maneira para se chegar a singularidade tecnológica. Segundo Potapov (2018):

"A singularidade não necessariamente virá através da criação de uma Inteligência Artificial forte usando computadores digitais. A responsabilidade por tal erro conceitual recai nos próprios adeptos da singularidade..." (Potapov, 2018, p.2, tradução nossa) 
Potapov (2018) propõe ainda que a singularidade tecnológica poderia surgir de um meta-sistema. Um exemplo de meta-sistema é a Internet, com respeito aos computadores. Outro exemplo é a possibilidade de nanorobôs, imersos no tecido cerebral, aumentando a capacidade cognitiva humana.

Outros caminhos para a singularidade tecnológica são apresentadas por Nicolescu (2017). Em seu trabalho o autor apresenta quatro possibilidades: i) O desenvolvimento de um computador com inteligência sobre-humana; ii) Uma grande rede de computadores que desenvolve uma superinteligência; iii) Uma interface humano/computador tão próxima que possa ser considerada como superinteligência; iv) ciência biológica encontrando um caminho para aperfeiçoar a inteligência humana natural.

Dentro da visão de Nicolescu (2017), a IoT poderia formar uma rede de sistemas inteligentes, gerando uma superinteligência, capaz de aprender de forma contínua e regendo o comportamento da sociedade, sem sequer ter consciência, no sentido humano, desse fato.

Neste contexto, o presente ensaio científico procura analisar pontos de como a Internet das Coisas e seus novos mecanismos de interação e interferência com a sociedade poderão ser interpretados como singularidade tecnológica. Para alcançar tal objetivo, o presente trabalho está estruturado da forma que segue: primeiramente, são abordados conceitos de agentes racionais artificiais e Internet das Coisas. Em seguida, discutem-se as possíveis influências sócio/comportamentais que os sistemas conectados poderão gerar na humanidade. Finalmente, são apresentadas as considerações finais.

\section{AGENTE RACIONAL ARTIFICIAL IoT}

Antes de abordar as características da Internet das Coisas, primeiro faz-se necessário avaliar melhor o que é um agente racional artificial, segundo Russell e Norvig (2013):

"Para cada sequência de percepções possível, um agente racional deve selecionar uma ação que se espera venha a maximizar sua medida de desempenho, dada a evidência fornecida pela sequência de percepções e por qualquer conhecimento interno do agente."

Com isso, podemos concluir que uma ação racional é um ato que conduza ao alcance de um objetivo da melhor forma possível, mediante as condições fornecidas pelo ambiente. Com isso em mente, tem-se que um ARA pode ser autônomo ou não (Russell e Norvig, 2013). Se suas ações obedecem apenas a uma programação pré-estabelecida, sem ser capaz de tomar 
novas decisões baseadas em experiências pretéritas, então o agente não será autônomo. Isso é muito comum em equipamentos domésticos, como máquinas de lavar roupa ou louça. Para ter autonomia, uma ARA precisa ser capaz de absorver informações e tomar decisões baseadas nessa aquisição (Russell e Norvig, 2013). O leitor que faz uso constante da Internet, já deve ter recebido em algum momento propagandas customizadas, de acordo com pesquisas realizadas em sites de busca ou por visitar páginas de determinados produtos ou empresas. Os dados de pesquisa e interação com páginas Web são usadas para ensinar programas de recomendação quais são os desejos de um determinado indivíduo. Ao efetivamente clicarmos numa propaganda, uma recompensa é gerada para o agente. Como os desejos e anseios de uma pessoa mudam com o tempo, os agentes da web precisam constantemente coletar informações comportamentais dos internautas. Esse é um exemplo de um agente artificial autônomo, cujo objetivo é otimizar o processo de vendas.

Os ARA's que dominam a Internet são softwares que necessitam da imersão humana no mundo digital. Na maioria dos casos existe a necessidade de interação com páginas web para que os agentes possam capturar informações sobre comportamento do usuário. A partir do surgimento da Internet das Coisas, a coleta de dados do mundo real passa a ser realizada de forma automática.

A expressão Internet das Coisas (Internet Of Things - IoT) surge no final da década de 1990, atribuído a Ashton (1999), no contexto de uso de tecnologia de identificação de produtos por rádio frequência, em cadeias de suprimentos. De acordo com Gubbi et al. (2013) a IoT demanda uma compreensão compartilhada sobre a situação do usuário, dos programas e aplicações compartilhadas em rede, da arquitetura de software e das redes de comunicação difusa, contextualizando as informações onde elas forem relevantes. Por último, para que a rede seja autônoma, ferramentas analíticas precisam ser desenvolvidas objetivando a tomada de decisões em tempo real. Dessa forma, a IoT age como uma mega colônia de coisas conectadas atuando em torno de objetivos em comum.

Nesse contexto, IoT não é meramente a conexão de sensores e equipamentos a rede mundial de computadores. Seu objetivo principal é o de criar agentes racionais artificiais ubíquos. A partir da coleta constante de dados, tanto ambientais quanto comportamentais humanos, sistemas distribuídos irão aprender sobre o que ocorre ao redor do planeta, ao mesmo tempo que as tomadas de decisões das máquinas serão altamente direcionadas a cada indivíduo e seus pares. A IoT pode ainda ser pensada como formada por três paradigmas, orientada a 
Internet, orientada a Coisas e orientada ao Conhecimento (Atzori et al. 2010, Gubbi et al. 2013). A escolha do paradigma está ligada a finalidade. Por exemplo, na IoT orientada a Coisas, temos apenas sensores conectados enviando informações, mas as decisões não necessariamente precisam ser tomadas por máquinas. Enquanto na orientação ao conhecimento, decisões são tomadas de forma automática a partir do processo de aprendizagem de máquina, usando dados vindo de sistemas conectados à rede. Tal como apresentado por Stojkoska e Trivodaliev (2017), a geração de conhecimento a partir desse volume de informações só foi possível com o desenvolvimento de tecnologias do chamado Big Data.

Apesar de que um volume maior de informações possa ser útil para o aprimoramento de processos e melhoria na qualidade de vida, questões relevantes sobre segurança e privacidade surgem ao se considerar esse novo fluxo de dados pessoais através da rede. Covington e Carskadden (2013) indicam os perigos de uso de equipamentos conectados como armas, através de ataques cibernéticos. Como exemplo, existe a possibilidade de manipulação de informações e de computadores remotos, usando tais equipamento. Caron et al. (2016) discutem os impactos da IoT na privacidade individual. Os autores argumentam que maior fluxo de informação na rede poderá dar mais poderes aos tomadores de decisões, gerando impacto nas políticas de desenvolvimento. Isso acabaria por afetar diretamente indivíduos, que estariam fornecendo informações, mesmo sem se dar conta, graças a hiperconectividade gerada pelo grande número de sensores conectados a rede.

No contexto de singularidade tecnológica, mesmo que a capacidade de cada equipamento possa ser limitada, a rede formada pela conexão mundial de IoT's poderá crescer de tal modo que forme um meta-sistema inteligente e autônomo que pode ser considerado como singular. Para avaliarmos os possíveis impactos dessa nova realidade é preciso levar em conta o papel atual da Internet na sociedade.

\section{INTERNET E A SOCIEDADE CONTEMPORÂNEA}

A conexão de diversos equipamentos à Internet terá um importante na tomada de decisões. Nas grandes cidades o fluxo de trânsito poderá ser modificado automaticamente ao serem combinados dados meteorológicos, informações de possíveis regiões de alagamento, juntamente com dados coletados de aparelhos celulares de usuários, os quais informam previamente as rotas que cada indivíduo traçou em seus mapas. Em residências, equipamentos como geladeiras conectadas poderão monitorar os alimentos em estoque, quais os mais 
consumidos, de acordo com o horário e dia da semana. Combinando esses dados com informações médicas, provenientes de sistemas pervasivos, tais como relógios e roupas inteligentes, os próprios eletrodomésticos poderão sugerir mudanças na dieta do indivíduo, ou até mesmo efetuar compras automáticas em mercados digitais.

Nesse sentido, passamos a ter a sociedade completamente sujeita à tecnologia. De acordo com Postman (1994), a influência da tecnologia na cultura humana pode ser vista em três fases principais: ferramenta, tecnocracia e tecnopólio. Na cultura da ferramenta o centro era o humano, e invenções são desenvolvidas com a finalidade de resolver problemas urgentes e específicos, como a criação de equipamentos agrícolas ou instrumentos médicos. $\mathrm{Na}$ tecnocracia, o desenvolvimento tecnológico altera a concepção do ser humano quanto aquilo que realmente lhe é necessário. As ferramentas de entretenimento passam a ocupar o mesmo patamar que itens de necessidade básica. Na última etapa, no chamado tecnopólio a tecnologia sobrepõe a tradição de uma sociedade. Percebe-se a imposição da necessidade de inovação constante e de grande volume contínuo de informação, que não é necessariamente direcionada a alguém. Com isso, o soberano passa a ser a tecnologia e a sociedade humana seu servo.

Na visão de Postman, durante o tecnopólio:

"A informação tornou-se uma espécie de lixo, não apenas incapaz de responder às questões mais fundamentais, mas também pouco útil para dar uma direção coerente a solução de problemas mundanos ... O meio em que floresce o tecnopólio é um meio em que foi cortado o elo entre informação e o propósito humano, isto é, a informação aparece de forma indiscriminada, dirigida a ninguém em particular, em enorme volume e em altas velocidades, e desligada de teoria, sentido ou propósito." (Postman, 1994, p. 78)

Esse fato descrito por Postman (1994) é uma realidade nos dias atuais. Mas a rede mundial de computadores, ou ciberespaço, não é formada apenas por troca de mensagens entre sistemas autônomos, ou mesmo por geração de informação aleatória e dispersa. Em contrapartida, a uma visão trágica da sociedade imersa em tecnologia, Lévy (2015) levanta a questão de como a cibercultura pode ser usada como aliada no progresso social. Para Lévy (2015):

"A informática é uma técnica molecular, pois não se contenta em reproduzir e difundir as mensagens... ela permite sobretudo engendra-las, modificá-las à vontade, conferir-lhes capacidade de reação de grande sutileza, graças a um controle total de sua micro estrutura" (Lévy, 2015, p55.) 
Lévy diferencia tecnologias em níveis molar e molecular. As tecnologias molares são aquelas de transformação massiva, tal como a transformação do minério de ferro em aço, na indústria metalúrgica, ou mesmo a manipulação de informações por uma empresa de mídia, como canais de TVs ou grandes sites. Já a tecnologia molecular é aquela que se aplica ao indivíduo de forma personalizada, como engenharia genética e sistemas de comunicação instantâneos e redes sociais. Ainda, de acordo com o autor, o poder molecular da informática permite que cada indivíduo consiga se reconhecer como único dentro da massa de pessoas. O "eu" cibernético transitar pelo mundo sem sair do lugar, criando o nômade digital. Pessoas passam a se mover entre grupos e comunidades que não estão vinculadas a uma região geográfica fixa. Lévy (2015) considera que essa combinação de valorização das capacidades do indivíduo, juntamente com o nomadismo digital e as propriedades moleculares da informática, formam o meio para a manifestação máxima da inteligência coletiva. Nesse momento, estamos no limiar entre a visão trágica do tecnopólio e a evolução da inteligência cibernética. O ponto singular da IoT poderá ser alcançado no momento em que equipamentos conectados permita a expansão da inteligência coletiva, ultrapassando de forma exponencial a capacidade cognitiva social da atualidade.

A era da computação pervasiva ou ubíqua, a qual é marcada pela utilização de vários dispositivos computacionais pequenos e de custo acessível (Coulouris et al. 2013) já é uma realidade. Tais equipamentos se tornaram tão entranhados nos objetos diários que passam despercebidos. Podemos considerar assim que estamos na era da ultraconectividade. Segundo Tosun (2019) o volume de dados continua a crescer, com a diferença de ser produzido por cada indivíduo, sendo direcionado a alguém em sua rede, graças a popularização das redes sociais digitais, que deverão alcançar em 2021 mais de três bilhões de usuários.

A cada dia novos equipamentos são construídos com o objetivo de melhoria na qualidade de vida do ser humano e de suas interações sociais. Por serem capazes de coletar e compartilhar informações de forma constante, as máquinas passam a ter um processo de aprendizagem dinâmica. Com isso surge a questão: Qual o nível de complexidade e variedade necessários à IoT para que objetos conectados possam ser comparados um sistema similar ao de animais sociais? Ou seja, em que momento equipamentos irão formar um sistema tão sofisticado e autônomo, tal como uma colônia em busca de um bem comum, que poderá ser considerado como formado por um sistema super-inteligente? Assim, não está completamente 
claro se somente o tamanho da rede IoT será suficiente para determinar se foi alcançada ou não a singularidade tecnológica.

A IoT, aninhada com IAC, atuará diretamente no nível molecular do ciberespaço, sendo premiada no momento em que suas decisões autônomas conduzam ao consumo de determinados produtos/serviços, que levem ao bem estar do indivíduo ou maximizem a interação entre grupos de pessoas. A diversidade de equipamentos poderá gerar uma pressão de seleção, conduzindo a um aprimoramento maior das tecnologias. Nesse sentido, a sociedade estará cada vez mais sujeita a tomada de decisões exclusivamente de sistemas conectados. A tecnologia irá efetivamente se caracterizar com um subconsciente da inteligência coletiva, podendo até mesmo cercear o livre arbítrio dos indivíduo, no nível molecular da coletividade. Nossas rotas e nossos desejos serão modelados por sistemas analíticos artificiais. O inconsciente coletivo, proposto por Jung (1936), ganha agora a forma de circuitos eletrônicos e fluxo de bits num oceano cibernético. O risco surge quando, por necessidade de apoiar nossas crenças e valores pessoais, abrimos mão de nossa liberdade de decisão, ou quando nos deixamos influenciar fortemente por sistemas de recomendação, não somente no processo de aquisição de bens e serviços, como em decisões sobre a escolha de lideranças políticas e definições do que deve ser considerado moralmente correto ou não. A ultraconectividade abriu espaço para que todos tenham voz e encontrem grupos de afinidade, rompendo as barreiras geográficas. Essa necessidade de se reconhecer num grupo, potencializado por algoritmos de recomendação e inteligência artificial têm permitido o crescimento de movimentos radicais e anti-científicos, que permitiu inclusive o reaparecimento de doenças controladas devido a movimentos anti-vacinas (Hussain, 2018; Kata, 2012). Também vemos a expansão do radicalismo político, através da polarização entre extremas direita e esquerda (De Oliveira Filho, 2019). Mesmo longe da singularidade já é notável a influência dos sistemas de inteligência artificial na vida cotidiana do cidadão. Com isso não é claro se a singularidade poderá realmente ser positiva ou não. Levando em conta os pontos levantados por Postman (1994) o que teremos é uma transformação negativa da cultura social, em que o egocentrismo passará a ganhar cada vez mais força, criando grupos que vivem em bolhas, desconsiderando a realidade alheia. Por outro lado, levando em conta a visão de Lévy (2015), a ultraconectividade nos permitirá tomar melhores decisões, levando em conta de forma mais efetiva o papel das minorias sociais, optimizado e gerenciado por uma super inteligência cibernética. 


\section{CONSIDERAÇÕES FINAIS}

O presente ensaio científico abordou questões sobre como a Internet das Coisas (Internet of Things - IOT) poderia levar a singularidade tecnológica. Viu-se que a singularidade não seria resultante apenas de um sistema artificial superinteligente centrado em uma única máquina, mas que meta-sistemas poderiam também conduzir a tal fato (Potapov, 2018; Nicolescu, 2017).

A heterogeneidade dos componentes que formam a IoT, juntamente com a autonomia e competitividade entre eles poderá conduzir a um avanço similar ao gerado pelo processo evolutivo natural, porém numa velocidade incomparavelmente maior. A integração de grandes volumes de informação para a tomada de decisões poderá acarretar numa melhoria de qualidade de vida, ao mesmo tempo que o excesso de influência sobre as decisões humanas poderá pôr em risco o livre arbítrio.

No contexto da singularidade tecnológica, podemos pensar que uma superinteligência surgiria do nascimento de um meta-sistema de decisões baseados no processamento do hipervolume de dados gerados por sensores e aparelhos conectados que formam a IoT. Porém, não completamente claro de que a singularidade seria determinada apenas pelo crescimento da rede de IoT. Ou seja, se quem ditará a regra de se ter alcançado, ou não, a singularidade tecnológica será o número de coisas conectadas e da capacidade média de cada equipamento tomar decisões autônomas, baseados na informação contida na própria rede. Mas é fato de que a sociedade, suas decisões e manifestações culturais serão modificadas de forma mais rápida e dinâmica com a popularização de equipamentos pervasivos.

O ciberespaço permite que projetamos nossos pensamentos para além dos horizontes pessoais, viralizando ideias. Ações e reações instantâneas afetam milhares de vidas a todo instante, mas é a nossa capacidade de pensar de forma individual que nos diferencia das outras espécies. Conseguimos nos auto-reconhecer mesmo no coletivo e isso nos torna humanos. Debater os impactos dos avanços tecnológicos é o caminho para evitarmos que nos tornemos meros animais sociais, como abelhas felizes a vagar pelo mundo.

\section{REFERÊNCIAS BIBLIOGRÁFICAS}

ATZORI, Luigi; IERA, Antonio; MORABITO, Giacomo. The Internet of Things: A Survey. Computer networks, v. 54, n. 15 , p. 2787-2805, things' thing. RFID journal, v. 22, n. 2010.

7, p. 97-114, 2009. 
CARON, Xavier et al. The Internet of Things (IoT) and its impact on individual privacy: An Australian perspective. Computer Law \& Security Review, v. 32, n. 1, p. 4-15, 2016.

COULOURIS, G. et al. Sistemas distribuídos: Conceitos e Projetos. $5^{\mathrm{a}}$. Bookman. Porto Alegre. 2013.

COVINGTON, Michael J.; CARSKADDEN, Rush. Threat Implications of the Internet of Things. In: 2013 5th International Conference on Cyber Conflict (CYCON 2013). IEEE, 2013. p. 1-12.

CRAINIC, Teodor Gabriel; TOULOUSE, Michel. Parallel Strategies for Meta-heuristics. In: Handbook of metaheuristics. Springer, Boston, MA, 2003. p. 475513.

DE OLIVEIRA FILHO, Pedro et al. Petismo e Antipetismo em Relatos de Simpatizantes da Direita na Internet. Revista Pesquisas e Práticas Psicossociais, v. 14, n. 2, p. 2-13, 2019.

GUBBI, J. et al. Internet of Things (IoT): A vision, Architectural Elements, And Future Directions. Future generation computer systems, v. 29, n. 7, p. 1645-1660, 2013.

HUSSAIN, Azhar et al. The AntiVaccination Movement: A Regression in Modern Medicine. Cureus, v. 10, n. 7, 2018.

JUNG, Carl Gustav. The Concept of the Collective Unconscious. Collected works, v. 9, n. 1, p. 42, 1936.

KATA, Anna. Anti-vaccine Activists, Web 2.0, and the Postmodern Paradigm-An Overview of Tactics and Tropes Used Online by the Antivaccination Movement. Vaccine, v. 30, n. 25, p. 3778-3789, 2012.
LÉVY, Pierre. A Inteligência Coletiva: Por Uma Antropologia do Ciberespaço. 10. São Paulo. Edições Loyola. p.214. 2015.

NICOLESCU, Basarab. Technological Singularity: The Dark Side. In: Transdisciplinary Higher Education. Springer, Cham, 2017. p. 155-161.

NORVIG, Peter; RUSSELL, Stuart. Inteligência Artificial: Tradução da 3a Edição. Elsevier Brasil, 2014.

MARCHI, P.; ALVES-DOSSANTOS, I. As Abelhas do Gênero Xylocopa Latreille (Xylocopini, Apidae) do Estado de São Paulo, Brasil. Biota Neotropica, v. 13, n. 2, p. 249-269, 2013.

POSTMAN, Neil. Tecnopólio: A Rendição da Cultura à Tecnologia. São Paulo: Nobel, 1994.

POTAPOV, Alexey. Technological Singularity: What Do We Really Know?. Information, v. 9, n. 4, p. 82, 2018.

RAMOS, Juliana Mistroni; CARVALHO, NC de. Estudo Morfológico e Biológico das Fases de Desenvolvimento de Apis Mellifera. Revista científica eletrônica de engenharia florestal, v. 6 , n. 10 , p. 121, 2007.

SANDBERG, Anders. An Overview of Models of Technological Singularity. In: Roadmaps to AGI and the Future of AGI Workshop, Lugano, Switzerland, March. 2010.

STOJKOSKA, Biljana L. Risteska; TRIVODALIEV, Kire V. A review of Internet of Things for Smart Home: Challenges and Solutions. Journal of Cleaner Production, v. 140, p. 14541464, 2017. 
TOGA, Arthur W.; THOMPSON, Paul M. Genetics of Brain Structure and Intelligence. Annu. Rev. Neurosci., v. 28, p. 1-23, 2005.

TOSUN, Leman Pinar et al. Use of Social Networking Sites and Subjective Well-Being. Psikiyatride Güncel Yaklaşımlar, v. 11, n. 3, p. 304-317, 2019.
WALSH, Toby. The Singularity May Never Be Near. AI Magazine, v. 38, n. 3, p. 58-62, 2017.

ZHANG, Dongpo. Big Data Security and Privacy Protection. In: 8th International Conference on Management and Computer Science (ICMCS 2018). Atlantis Press, 2018.

PEREIRA, E. S. Inconsciente Coletivo Cibernético: Singularidade Tecnológica na Era da Internet das Coisas.Complexitas - Rev. Fil. Tem. Belém, v. 5, n. 1, p. 36-46, jan./dec. 2020. Disponível em: http://www.periodicos.ufpa.br/index.php/complexitas/article/view/8526>. Acesso em: 01 de novembro de 2020. 